EUROPEAN ORGANIZATION FOR NUCLEAR RESEARCH

CERN-PPE/95-xxx

July 20,1995

\title{
An Improved Theoretical Prediction for the 2-Jet Rate in $\mathrm{e}^{+} \mathrm{e}^{-}$Annihilation
}

\author{
Günther Dissertori \\ Institute of Experimental Physics, Innsbruck, Austria \\ Michael Schmelling \\ CERN, Geneva, Switzerland
}

\begin{abstract}
We show that an Ansatz to resum all leading and next-to-leading logarithms in the theoretical prediction for the 2-jet rate in $e^{+} e^{-} \rightarrow$ hadrons, where jets are defined with the $k_{t}$-algorithm, is consistent with a full $\mathcal{O}\left(\alpha_{s}^{2}\right)$ calculation done by Monte Carlo integration. From the asymptotic behaviour of the full $\mathcal{O}\left(\alpha_{s}^{2}\right)$ calculation we extract the subleading coefficient $G_{21}$ and the constant $C_{2}$.
\end{abstract}

submitted to Phys.Lett.B 


\section{Introduction}

The study of global event shape variables in $e^{+} e^{-}$annihilation is one of the most precise methods to determine the strong coupling constant $\alpha_{s}$. Here the very accurate data obtained at LEP have led to a situation, where the error of $\alpha_{s}$ is dominated by the theoretical uncertainties [1], related to uncalculated higher order terms in the perturbative expansion or to non-perturbative hadronization effects. For $\alpha_{s}$-measurements based on the 2 -jet rate $R_{2}$ the theoretical error comes mainly from the perturbative sector, i.e. any improvement in the perturbative prediction would lead to a better measurement of $\alpha_{s}$.

At present, full perturbative calculations for any infrared and collinear safe event shape variable exist only up to $\mathcal{O}\left(\alpha_{s}{ }^{2}\right)[2]$. For some variables also leading and next-to-leading logarithms have been resummed to all orders in $\alpha_{s}[3,4,5,6]$. See [7] for a detailed description of these resummation techniques, which use the coherent branching algorithm to next-to-leading logarithmic accuracy.

If calculated with the $k_{t}$-algorithm $[8,9,6]$ based on the resolution parameter

$$
y_{i j}=\frac{2 \min \left(E_{i}^{2}, E_{j}^{2}\right)}{E_{c m}^{2}}\left(1-\cos \Theta_{i j}\right),
$$

the perturbative prediction for $R_{2}$ is known to exponentiate, which allows to resum leading and next-to-leading logarithms $L=-\ln \left(y_{3}\right)$, where $y_{3}$ is the resolution parameter where the event undergoes the transition from a 3 -jet to a 2 -jet event. Theoretical calculations $[6]$ are available, which resum all leading and part of the next-to-leading logarithms. This work describes an Ansatz for the resummed prediction of $R_{2}$, motivated by the exponentiation behaviour of similar terms for other variables $[3,4,5]$, which tries to include the missing next-to-leading logarithmic contributions.

\section{The Theoretical Framework}

In order to simplify the expressions describing the theoretical prediction for $R_{2}$, it is convenient to introduce the colour factor ratios $f_{A}$ and $f_{T}$ and to redefine the strong coupling constant by absorbing into it a factor of $C_{F} / 2 \pi$. This leads to the definitions:

$$
f_{A}=\frac{C_{A}}{C_{F}} \quad, \quad f_{T}=N_{f} \frac{T_{F}}{C_{F}} \quad \text { and } \quad \bar{\alpha}_{s}=\frac{\alpha_{s} C_{F}}{2 \pi} .
$$

For the following the number of active quark flavours is $N_{f}=5$. The QCD values for the colour factors are $C_{F}=4 / 3, C_{A}=3$ and $T_{F}=1 / 2$.

According to general theorems $[10]$ the perturbative prediction for the cumulative cross section of any event shape variable $y$, which vanishes in the limit of perfect 2-jet topologies, can be expressed in the form,

$$
R(L)=\frac{\sigma(-\ln (y)>L)}{\sigma_{\text {tot }}}=\left(1+\sum_{i=1}^{\infty} C_{i} \bar{\alpha}_{s}^{i}\right) \exp \left(\sum_{n=1}^{\infty} \sum_{m=1}^{2 n} G_{n m} \bar{\alpha}_{s}^{n} L^{m}\right)+\sum_{k=1}^{\infty} \bar{\alpha}_{s}^{k} D_{k}(L),
$$

where for clarity any explicit dependence on the renormalization scale $\mu^{2}$ is suppressed. The $D_{k}(L)$ are regular functions which vanish in the limit $L \rightarrow \infty$. The terms in the double sum are 
classified as leading logarithms (LL) for $m>n$, next-to-leading logarithms (NLL) for $m=n$ and sub-leading for $m<n$. Exponentiation of the perturbative prediction means $G_{n m}=0$ for $m>n+1$. In this case one can write

$$
\sum_{n=1}^{\infty} \sum_{m=1}^{n+1} G_{n m} \bar{\alpha}_{s}^{n} L^{m}=\ln \Sigma\left(\bar{\alpha}_{s}, L\right)=L g_{1}\left(\bar{\alpha}_{s} L\right)+g_{2}\left(\bar{\alpha}_{s} L\right)+\cdots
$$

Thus the function $L g_{1}\left(\bar{\alpha}_{s} L\right)$ resums all the LL contributions $\bar{\alpha}_{s}^{n} L^{n+1}, g_{2}\left(\bar{\alpha}_{s} L\right)$ contains the NLL terms $\bar{\alpha}_{s}^{n} L^{n}$ and the remainder the sub-leading corrections.

The resummed expression for $\ln \Sigma_{R_{2}\left(y_{3}\right)}$, where $R_{2}\left(y_{3}\right)$ is the 2-jet rate as function of the cut-off $y_{3}$ is given in $[6]$ as

$$
\ln \Sigma_{R_{2}\left(y_{3}\right)}=-2 \int_{s y_{3}}^{s} \frac{d q^{2}}{q^{2}} \bar{\alpha}_{s}\left(q^{2}\right)\left(\ln \frac{s}{q^{2}}-\frac{3}{2}\right) .
$$

The explicit dependence on the renormalization scale $\mu^{2}$ can be obtained by expanding $\bar{\alpha}_{s}\left(q^{2}\right)$ in terms of $\bar{\alpha}_{s}\left(\mu^{2}\right)$, using the solution of the renormalization group equation given in [11]

$$
\bar{\alpha}_{s}\left(q^{2}\right)=\frac{\bar{\alpha}_{s}\left(\mu^{2}\right)}{w}\left(1-\frac{b_{1}}{b_{0}} \frac{\bar{\alpha}_{s}\left(\mu^{2}\right)}{w} \ln w\right) \quad, \quad w=1-b_{0} \bar{\alpha}_{s}\left(\mu^{2}\right) \ln \frac{\mu^{2}}{q^{2}}
$$

with

$$
b_{0}=\frac{11}{6} f_{A}-\frac{2}{3} f_{T} \quad \text { and } \quad b_{1}=\frac{17}{6} f_{A}^{2}-\left(\frac{5}{3} f_{A}+1\right) f_{T}
$$

One of the main ingredients of the coherent branching algorithm is the Altarelli-Parisi (AP) splitting function $P_{q q}\left(\bar{\alpha}_{s}, z\right)[12]$, which describes the parton branching $q \rightarrow q g$ as function of the longitudinal momentum fraction $z$. In the infrared limit it is given by

$$
P_{q q}\left(\bar{\alpha}_{s}, z \rightarrow 1\right)=\frac{2}{1-z}\left(\bar{\alpha}_{s}+\bar{\alpha}_{s}^{2} K\right)
$$

where

$$
K=f_{A}\left(\frac{67}{18}-\frac{\pi^{2}}{6}\right)-f_{T} \frac{10}{9}=f_{A} K_{A}+f_{T} K_{T}
$$

For the calculation in [6] only the first order part of Eq.(8) has been used, which gives rise to the terms $\bar{\alpha}_{s}\left(q^{2}\right) \ln \left(s / q^{2}\right)$ under the integral Eq.(5). The term $\propto 3 / 2$ in Eq. (5) comes from the $(+)$-regularization [13] of the AP splitting function.

Motivated by the fact that for other event shape variables also the next-to-leading part of the AP splitting function exponentiates, we assume that the same holds for the 2-jet rate. The functional form of Eq.(8) suggests the following Ansatz:

$$
\ln \Sigma_{R_{2}\left(y_{3}\right)}=-2 \int_{s y_{3}}^{s} \frac{d q^{2}}{q^{2}}\left\{\bar{\alpha}_{s}\left(q^{2}\right)\left(\ln \frac{s}{q^{2}}-\frac{3}{2}\right)+\bar{\alpha}_{s}^{2}\left(q^{2}\right) K \ln \frac{s}{q^{2}}\right\} .
$$

Doing the integration and dropping all subleading terms, the result can be expressed as

$$
\ln \Sigma_{R_{2}\left(y_{3}\right)}=L g_{1}(x)+g_{2}(x)+x^{2} g_{1}^{\prime}(x) \ln \frac{\mu^{2}}{s}
$$


with $L=-\ln \left(y_{3}\right)$ and $x=\bar{\alpha}_{s}\left(\mu^{2}\right) b_{0} L$. The prime in $g_{1}^{\prime}(x)$ stands for derivative with respect to the given argument. The functions $g_{1}(x)$ and $g_{2}(x)$ are:

$$
\begin{gathered}
g_{1}(x)=\frac{2}{b_{0}}\left(1+\frac{\ln (1-x)}{x}\right) \\
g_{2}(x)=-\frac{3}{b_{0}} \ln (1-x)-\frac{2 K}{b_{0}^{2}}\left(\frac{x}{1-x}+\ln (1-x)\right)+\frac{2 b_{1}}{b_{0}^{3}}\left(\frac{x+\ln (1-x)}{1-x}+\frac{1}{2} \ln ^{2}(1-x)\right)
\end{gathered}
$$

Expanding the above functions in powers of $\bar{\alpha}_{s}$ one sees that the terms proportional to $K$ only contribute to $\mathcal{O}\left(\bar{\alpha}_{s}^{2}\right)$. The additional terms are proportional to the colour factors $C_{A}$ and $T_{F}$, expressing the fact that we have included missing NLL terms coming from higher order corrections to the fundamental $q \rightarrow q g$ branching. The renormalization scale dependence of $\ln \Sigma_{R_{2}\left(y_{3}\right)}$ is entirely determined by the LL function $g_{1}(x)$, i.e. it is independent of $K$. It is worth noting [14] that the additional NLL terms proportional to $K$ could also be generated in the original formula [6] by choosing the renormalization scale $\ln \mu^{2} / s=K / b_{0}$.

\section{Comparison with the Full $\mathcal{O}\left(\alpha_{\mathrm{s}}^{2}\right)$ Calculation}

In the following the renormalization scale will be set to $\mu^{2}=s$. The leading coefficients $G_{n m}$ of the full perturbative prediction Eq.(3) can be read off by expanding the result for $\ln \Sigma_{R_{2}\left(y_{3}\right)}$ in powers of $x$ :

$$
\ln \Sigma_{R_{2}\left(y_{3}\right)}=\frac{x}{b_{0}}(3-L)+\left(\frac{x}{b_{0}}\right)^{2}\left(\frac{3 b_{0}}{2}-K-\frac{2 b_{0}}{3} L\right)+\mathcal{O}\left(x^{3}\right)
$$

From this the leading coefficients are read off as $G_{12}=-1, G_{11}=3, G_{23}=-2 b_{0} / 3$ and $G_{22}=3 b_{0} / 2-K$. The coefficient $C_{1}$ has been obtained in [9] by computing the 3-jet fraction $R_{3}(L)$ in lowest order ${ }^{1}$. There one finds

$$
C_{1}=-6 \ln 2-\frac{5}{2}+\frac{\pi^{2}}{6}
$$

The exact expressions for $D_{2}(L), G_{21}, C_{2}$ are not known. The function $D_{1}(L)$ can be extracted from the integral of the analytical first order result given in the appendix.

For the comparison with the Monte Carlo integration of the second order matrix element it is more convenient to use the differential cross section

$$
\frac{1}{\sigma_{0}} \frac{d \sigma}{d L}=-\frac{\sigma_{h a d}}{\sigma_{0}} \frac{d R_{2}}{d L}
$$

Here $\sigma_{0}$ is the Born cross section for $e^{+} e^{-} \rightarrow q \bar{q}$, which is related to the full hadronic cross section $\sigma_{\text {had }}$ by

$$
\frac{\sigma_{h a d}}{\sigma_{0}}=1+\frac{3}{2} \bar{\alpha}_{s}+\mathcal{O}\left(\bar{\alpha}_{s}^{2}\right)
$$

\footnotetext{
${ }^{1}$ Note that formula (8) in reference [9] can not be correct because the 3 -jet fraction does not vanish at the phase space boundary $y_{3}=1 / 3$. However, it has been checked numerically that the asymptotic behaviour $L \rightarrow \infty$ and thus the constant $C_{1}$ is reproduced correctly.
} 
By inserting the values for the coefficients $G_{n m}$ and separating the individual colour factor contributions one finally gets:

$$
\begin{aligned}
\frac{1}{\sigma_{0}} \frac{d \sigma}{d L} & =\bar{\alpha}_{s} \quad\left[2 L-3+S_{1}(L)\right] \\
& +\bar{\alpha}_{s}^{2}\left[-2 L^{3}+9 L^{2}-\left(6-2 C_{1}\right) L-G_{21, F}+S_{F}(L)-3 C_{1}-\frac{9}{2}+\frac{3}{2} S_{1}(L)\right] \\
& +\bar{\alpha}_{s}^{2} f_{A}\left[\frac{11}{3} L^{2}-\left(\frac{11}{2}-2 K_{A}\right) L-G_{21, A}+S_{A}(L)\right] \\
& +\bar{\alpha}_{s}^{2} f_{T}\left[-\frac{4}{3} L^{2}+\left(2+2 K_{T}\right) L-G_{21, T}+S_{T}(L)\right]+\mathcal{O}\left(\bar{\alpha}_{s}^{3}\right)
\end{aligned}
$$

The colour factor decomposition of $K$ is given in Eq.(9). Similarly one has $G_{21}=G_{21, F}+$ $f_{A} G_{21, A}+f_{T} G_{21, T}$. The functions $S_{*}(L), *=F, A, T$, are defined by derivatives of the functions $D_{1}$ and $D_{2}$ introduced in Eq. (3) as $-D_{1}^{\prime}(L)=S_{1}(L)$ and $-D_{2}^{\prime}(L)=S_{F}(L)+f_{A} S_{A}(L)+f_{T} S_{T}(L)$.

\subsection{The Numerical Calculation}

The differential cross section can be written in the form

$$
\frac{1}{\sigma_{0}} \frac{d \sigma}{d L}=\bar{\alpha}_{s} a(L)+\bar{\alpha}_{s}^{2}\left[b_{F}(L)+f_{A} b_{A}(L)+f_{T} b_{T}(L)\right]
$$

We have used a modified version of the Monte Carlo program EVENT [15] to integrate the second order QCD ERT matrix elements [16] in order to calculate the $a$ and $b$ coefficients. The program has been changed with respect to the user interface, the random number generator and to allow to get the coefficients of each colour factor separately. In total we have generated $33.6214 \times 10^{9}$ events and stored the functions $a, b$ in bins of width $\Delta L=0.2$. The results for the E-scheme are tabulated in Tab. 2 for the $L$ range over which the calculations are stable. The integration was done also for the E0, P and P0-schemes [17]. Tabulated coefficients for these schemes can be obtained from the authors. The first order prediction $a(L)$ is scheme independent. Figure 1 shows how the second order coefficients for the different schemes approach each other at large $L$. With exception of $b_{F}$ for the P0-scheme all curves come together at large $L$, indicating that up to $\mathcal{O}\left(\bar{\alpha}_{s}^{2}\right)$ also the subleading coefficients $G_{21, A}$ and $G_{21, T}$ are independent of the recombination scheme. The following is based only on the results obtained for the E-scheme.

The functions $a, b$ contain all LL, NLL and subleading terms correctly up to $\mathrm{O}\left(\bar{\alpha}_{s}^{2}\right)$. Thus a comparison of the numerical calculation with the expansion Eq.(18) at high $L$ allows to test the Ansatz Eq.(10). In this region the two calculations should only differ by a constant plus a contribution which vanishes asymptotically.

The function a was compared with the full analytical calculation given in the appendix. The calculations agree with each other over the whole $L$ range (Fig. 2a), giving confidence in the precision of the numerical integration. The functions $b_{*}$ follow the expected behaviour at large $L$ as can be seen from Figs. 2b, 2c and 2d. At low $L$ the influence of subleading terms is clearly visible. In the case of the coefficients $b_{A}(L)$ and $b_{T}(L)$ also the effect of setting $K=0$ is shown, demonstrating the need of the additional term in the Ansatz Eq.(10) and the sensitivity to this NLL contribution. 


\subsection{Study of the Leading-Order non-Logarithmic Terms}

In the limit of large $L$ the result given in the appendix for the differential 2-jet rate can be expanded to yield

$$
\frac{1}{\sigma_{0}} \frac{d \sigma^{1 s t}}{d L}=\bar{\alpha}_{s}\left(2 L-3+A e^{-L / 2}+B e^{-L}+\cdots\right)
$$

with

$$
A=4 \sqrt{2}+\ln (3-2 \sqrt{2}) \quad \text { and } \quad B=18 \ln 2-1-4 L \quad .
$$

The LL and NLL terms are the same as those in Eq.(18), and one finds the non-logarithmic terms $S_{1}(L)$ to behave like

$$
S_{1}(L)=\frac{1}{\sigma_{0}} \frac{d \sigma^{1 s t}}{d L}-\bar{\alpha}_{s}(2 L-3)=\bar{\alpha}_{s}\left(A e^{-L / 2}+B e^{-L}+\cdots\right)
$$

The inset in Fig. 2 shows the relative contribution of $S_{1}(L)$ with respect to the full first order prediction as function of $L$. It can be seen that this contribution falls below the $1 \%$ level only at $L \approx 8$, which corresponds to $y_{3} \approx 3.35 \times 10^{-4}$.

\subsection{Determination of the Coefficients $\mathrm{G}_{21}$ and $\mathrm{C}_{2}$}

Having subtracted all the known terms as given in Eq.(18) from the numerically obtained coefficients $b_{*}$, the resulting differences $\Delta b_{*}$ are plotted in Fig. 3. Assuming the asymptotic behaviour of all subleading functions $S_{*}(L)$ to be the same as for $S_{1}(L)$, we have fitted functions

$$
a_{0}+a_{1} e^{-L / 2}+a_{2} e^{-L}
$$

separately to each $\Delta b_{*}$ in the asymptotic region. This allows to extract the subleading coefficients $G_{21, *}$ for each colour factor. The results are listed in Tab. 1.

\begin{tabular}{|c|rcccc|c|}
\hline$*$ & $G_{21, *}$ & \pm & stat & \pm & syst & Fit Range in $L$ \\
\hline$F$ & -16.081 & \pm & 0.277 & \pm & 1.304 & $5.6 \rightarrow 10.0$ \\
$A$ & -4.551 & \pm & 0.081 & \pm & $\mathbf{0 . 2 9 6}$ & $5.6 \rightarrow 10.0$ \\
$T$ & $\mathbf{0 . 9 0 0}$ & \pm & $\mathbf{0 . 0 1 8}$ & \pm & $\mathbf{0 . 0 8 7}$ & $7.4 \rightarrow 12.0$ \\
\hline
\end{tabular}

Table 1: Fitted values for the subleading coefficients $G_{21, *}$.

The determination of the coefficients $G_{21, *}$ was done as follows: Fixing the upper limit of the fit range and decreasing the lower limit as long as the $\chi^{2} / N_{d f}$ is near to one, we first fit only the parameter $a_{0}$. The other two are set to zero. The result for $G_{21, *}$ obtained with the largest fit range is retained. Then the procedure is repeated with $a_{1}$ as additional free parameter and finally with all three varying freely. As central value the result $G_{21, *}$ is chosen, which was obtained with the $a_{0}$ and $a_{1}$ allowed to vary and $a_{2}$ set to zero. The statistical error reflects the finite Monte Carlo statistics, the systematic error is the larger of the differences to the other variants. Combining the coefficients for the individual different colour factors yields for QCD

$$
G_{21}=-24.633 \pm 1.509 \quad \text {. }
$$


Using the fitted value of $G_{21}$, the procedure described above is applied to the difference between the numerically obtained 2-jet rate $R_{2}(L)$ and the second order expansion of Eq.(3). Taking the correlations between the bins of the $R_{2}$-distribution into account we obtain the following result for the QCD coefficient $C_{2}$ :

$$
C_{2}=99.12 \pm 2.75+4.30\left(G_{21}-G_{21}^{v a r}\right)
$$

The third term parametrizes the change in $C_{2}$ when the central value $G_{21}$ is varied to $G_{21}^{v a r}$. Figure 4 illustrates the effect of adding $G_{21}$ and $C_{2}$ to the LL plus NLL prediction of the 2jet rate. The above results are consistent with another recent calculation [18], however, the errors given there are rather large and the possible effect of non-logarithmic contribution was neglected.

\section{$4 \quad$ A Fit for $\alpha_{\mathrm{s}}\left(\mathbf{M}_{\mathrm{Z}}^{2}\right)$}

The effect of the improved prediction on a measurement of the strong coupling constant was studied by applying the same procedure used in [11] to the $y_{3}$ distribution generated with the JETSET [19] Monte Carlo model. It turns out, that a measurement based on the improved theory lowers the central value of $\alpha_{s}$ by $60 \%$ of the theoretical uncertainty. The relative theoretical error, determined according to the prescription given in [11], remains constant at $\Delta \alpha_{s} / \alpha_{s}=0.038$.

Knowing the subleading term $G_{21}$ and the constant $C_{2}$ it is now also possible to apply the "intermediate" matching scheme as described in [11] to the case of the $y_{3}$ distribution. The results are very similar to the results obtained for the $\ln R$ scheme. The same observation was made in [11] for the event shape variables Thrust and Heavy Jet Mass.

\section{Summary}

We have shown that a new Ansatz for the resummed prediction for the 2-jet rate, based on the assumption that the infrared singular part of the next-to-leading AP splitting function $P_{q q}$ exponentiates, is consistent with a full $\mathcal{O}\left(\alpha_{s}{ }^{2}\right)$ calculation obtained from a high statistics Monte Carlo integration of the ERT matrix elements. Using this Ansatz we are able to extract the subleading coefficients by fitting the asymptotic tail of the numerical calculation. The results for the $\mathrm{E}-$ scheme are $G_{21}=-24.633 \pm 1.509$ and $C_{2}=99.12 \pm 2.75$, where $C_{2}$ is a function of $G_{21}$. The numerical values apply for a perturbative expansion in $\bar{\alpha}_{s}=\alpha_{s} C_{F} / 2 \pi$. An $\alpha_{s}$-measurement based on the new theoretical prediction and the same procedure as used in [11] lowers the central value of $\alpha_{s}$ by $60 \%$ of the theoretical error. The relative size of the theoretical uncertainty remains constant.

\section{Acknowledgements}

We would like to thank S. Catani, M. Seymour and B. Webber for many valuable discussions and suggestions. 


\section{Appendix: The Differential 2-Jet Rate in Leading Order}

The differential 2-jet rate in first order is obtained by calculating the integral

$$
\frac{d \sigma^{1 s t}}{d y_{3}}=\int d x_{q} d x_{\bar{q}} \frac{d^{2} \sigma}{d x_{q} d x_{\bar{q}}} \delta\left(y_{3}-\hat{y}_{3}\left(x_{q}, x_{\bar{q}}\right)\right)
$$

over the appropriate phase space, where the matrix element for $e^{+} e^{-} \rightarrow q \bar{q} g$ is [20]

$$
\frac{1}{\sigma_{0}} \frac{d^{2} \sigma}{d x_{q} d x_{\bar{q}}}=\bar{\alpha}_{s} \frac{x_{q}^{2}+x_{\bar{q}}^{2}}{\left(1-x_{q}\right)\left(1-x_{\bar{q}}\right)} .
$$

Here $x_{q}$ and $x_{\bar{q}}$ are the scaled energies of the quark and the anti-quark respectively, defined as $x=2 E / E_{C M}$. For energy ordered configurations $x_{1}>x_{2}>x_{3}$ the variable $\hat{y}_{3}$ determined by the $k_{t}$-algorithm is given by

$$
\hat{y}_{3}=\left(1-x_{1}\right) \frac{x_{3}}{x_{2}}
$$

One obtains:

$$
\frac{1}{\sigma_{0}} \frac{d \sigma^{1 s t}}{d y_{3}}=\bar{\alpha}_{s} f_{3}\left(y_{3}\right)
$$

with

$$
\begin{aligned}
f_{3}\left(y_{3}\right) & =-7+12 b-\frac{3}{y_{3}}+16 y_{3}-\frac{\left(1-y_{3}\right)\left(12 y_{3}-12 b y_{3}-5 y_{3}^{2}\right)}{\left(2-2 b-y_{3}\right)^{2}}+\frac{6-8 y_{3}+2 y_{3}^{2}}{1-b+y_{3}} \\
& +\frac{b^{2}+2 b y_{3}+4 y_{3}^{2}}{1-y_{3}}-\frac{4 \sqrt{y_{3}}}{1-y_{3}} \ln \left(\frac{1+\sqrt{y_{3}}}{1-\sqrt{y_{3}}}\right)-\frac{1}{\sqrt{y_{3}}} \frac{1+y_{3}}{1-y_{3}} \ln \left(\frac{2-2 b-\sqrt{y_{3}}}{2-2 b+\sqrt{y_{3}}}\right) \\
& +\frac{2}{\sqrt{y_{3}}} \ln \left(\frac{\sqrt{y_{3}}-1+b}{\sqrt{y_{3}}+1-b}\right)+2 \frac{1+y_{3}}{1-y_{3}} \ln \left(\frac{1-y_{3}}{2}\right)+\frac{2+y_{3}}{y_{3}} \ln \left(2 \frac{1-y_{3}}{2-b}\right) \\
& +\frac{6-10 y_{3}+8 y_{3}^{2}}{1-y_{3}} \ln \left(2 \frac{2-2 b-y_{3}}{1-y_{3}}\right)-\frac{4}{y_{3}\left(1-y_{3}\right)} \ln \left(\frac{y_{3}}{1-b}\right) \\
& -\frac{1+y_{3}}{1-y_{3}} \ln \left(2 b y_{3}-y_{3}\right)+\left(8-4 y_{3}\right) \ln \left(\frac{2 y_{3}}{1-b+y_{3}}\right)
\end{aligned}
$$

and $b=1+y_{3} / 4-\sqrt{y_{3} / 2+y_{3}^{2} / 16}$. 


\section{References}

[1] M. Schmelling, Physica Scripta 51 (1995) 683.

[2] Z. Kunszt, P. Nason, G. Marchesini and B.R. Webber, in "Z Physics at LEP1", CERN 89-08, vol. 1 , p. 373.

[3] S. Catani, G. Turnock, B.R. Webber and L. Trentadue, Phys. Lett. B263 (1991) 491.

[4] S. Catani, G. Turnock and B.R. Webber, Phys. Lett. B272 (1991) 368.

[5] S. Catani, G. Turnock and B.R. Webber, Phys. Lett. B295 (1992) 269.

[6] S. Catani, Y.L. Dokshitzer, M. Olsson, G. Turnock and B.R. Webber, Phys. Lett. B269 (1991) 432 .

[7] S. Catani, L. Trentadue, G. Turnock and B.R. Webber, CERN Preprint CERNTH.6640/92 (1992), Cavendish-HEP-91/11.

[8] Y.L. Dokshitzer, Contribution to the Workshop on Jets at LEP and HERA, Durham (1990).

[9] N. Brown, W.J. Stirling, Z. Phys. C53 (1992) 629

[10] F. Bloch, A. Nordsieck, Phys. Rev. 52 (1937) 54;

T. Kinoshita, J. Math. Phys. 3 (1962) 650;

T.D. Lee, M. Nauenberg, Phys. Rev. 133 (1964) 1549.

[11] ALEPH Collaboration, D. Decamp et al., Phys. Lett. B284 (1992) 163.

[12] G. Altarelli, G. Parisi, Nucl. Phys. B126 (1977) 298.

[13] G. Altarelli, Phys. Rep. 81 (1982) 1.

[14] S. Catani, G. Marchesini, B.R. Webber, Nucl. Phys. B349 (1991) 635.

[15] P. Nason, private communication.

[16] R.K. Ellis, D.A. Ross, A.E. Terrano, Nucl. Phys. B178 (1981) 421.

[17] S. Bethke, Z. Kunszt, D.E. Soper, W.J. Stirling, Nucl. Phys. B370 (1992) 310.

[18] G. Kramer and H. Spiessberger, Preprint DESY 95-097.

[19] T. Sjöstrand, Comp. Phys. Comm. 27 (1982) 243;

T. Sjöstrand, Comp. Phys. Comm. 28 (1983) 229;

T. Sjöstrand and M. Bengtson, Comp. Phys. Comm. 43 (1987) 367.

[20] J. Ellis, M.K. Gaillard and G.G. Ross, Nucl. Phys. B111 (1976) 253. 


\begin{tabular}{|c|c|c|c|c|}
\hline$L$ & $a \pm s t a t$ & $b_{F} \pm s t a t$ & $b_{A} \pm s t a t$ & $b_{T} \pm s t a t$ \\
\hline $1.0-1.2$ & $0.07762 \pm 0.00001$ & $-0.1044 \pm 0.0005$ & $0.4048 \pm 0.0003$ & $-0.26399 \pm 0.00004$ \\
\hline $1.2-1.4$ & $0.54700 \pm 0.00002$ & $1.6420 \pm 0.0011$ & $3.4988 \pm 0.0006$ & $-1.70748 \pm 0.00010$ \\
\hline $1.4-1.6$ & $0.98268 \pm 0.00003$ & $2.9449 \pm 0.0016$ & $6.4687 \pm 0.0008$ & $-3.09932 \pm 0.00015$ \\
\hline $1.6-1.8$ & $1.36284 \pm 0.00004$ & $3.7449 \pm 0.0021$ & $9.3027 \pm 0.0011$ & $-4.37929 \pm 0.00020$ \\
\hline $1.8-2.0$ & $1.71658 \pm 0.00004$ & $4.1990 \pm \mathbf{0 . 0 0 2 7}$ & $12.2312 \pm 0.0014$ & $-5.63718 \pm 0.00025$ \\
\hline $2.0-2.2$ & $2.05878 \pm 0.00005$ & $4.2715 \pm \mathbf{0 . 0 0 3 4}$ & $15.3390 \pm 0.0017$ & $-6.92511 \pm 0.00031$ \\
\hline $2.2-2.4$ & $2.39721 \pm 0.00006$ & $3.8738 \pm 0.0041$ & $18.6777 \pm 0.0021$ & $-8.27477 \pm 0.00037$ \\
\hline $2.4-2.6$ & $2.73576 \pm 0.00007$ & $2.9528 \pm 0.0050$ & $22.2672 \pm 0.0025$ & $-9.70000 \pm 0.00045$ \\
\hline $2.6-2.8$ & $3.07686 \pm 0.00009$ & $1.3971 \pm 0.0060$ & $26.1083 \pm 0.0030$ & $-11.21441 \pm 0.00053$ \\
\hline $2.8-3.0$ & $3.42154 \pm 0.00010$ & $-0.8065 \pm 0.0073$ & $30.2253 \pm 0.0036$ & $-12.82078 \pm 0.00062$ \\
\hline $3.0-3.2$ & $3.77003 \pm 0.00011$ & $-3.7322 \pm 0.0087$ & $34.5944 \pm 0.0043$ & $-14.52290 \pm 0.00072$ \\
\hline $3.2-3.4$ & $4.12234 \pm 0.00013$ & $-7.4886 \pm 0.0104$ & $39.2463 \pm 0.0051$ & $-16.32211 \pm 0.00083$ \\
\hline $3.4-3.6$ & $4.47856 \pm 0.00015$ & $-12.1418 \pm 0.0124$ & $44.1680 \pm \mathbf{0 . 0 0 6 0}$ & $-18.21902 \pm 0.00096$ \\
\hline $3.6-3.8$ & $4.83866 \pm 0.00017$ & $-17.7410 \pm 0.0148$ & $49.3479 \pm 0.0071$ & $-20.21973 \pm 0.00110$ \\
\hline $3.8-4.0$ & $5.20174 \pm 0.00019$ & $-24.4027 \pm 0.0177$ & $54.8269 \pm 0.0084$ & $-22.31478 \pm 0.00126$ \\
\hline $4.0-4.2$ & $5.56775 \pm 0.00021$ & $-32.2591 \pm 0.0212$ & $60.5459 \pm 0.0099$ & $-24.51455 \pm 0.00144$ \\
\hline $4.2-4.4$ & $5.93670 \pm 0.00024$ & $-41.2980 \pm 0.0254$ & $66.5443 \pm 0.0117$ & $-26.81524 \pm 0.00165$ \\
\hline $4.4-4.6$ & $6.30921 \pm 0.00027$ & $-51.6850 \pm 0.0304$ & $72.8360 \pm 0.0137$ & $-29.22286 \pm 0.00187$ \\
\hline $4.6-4.8$ & $6.68288 \pm 0.00030$ & $-63.4745 \pm 0.0365$ & $79.3570 \pm 0.0161$ & $-31.72610 \pm 0.00212$ \\
\hline $4.8-5.0$ & $7.05892 \pm 0.00033$ & $-76.8683 \pm 0.0440$ & $86.2544 \pm 0.0189$ & $-34.33061 \pm 0.00241$ \\
\hline $5.0-5.2$ & $7.43726 \pm 0.00037$ & $-91.8648 \pm 0.0530$ & $93.3017 \pm 0.0222$ & $-37.04959 \pm 0.00272$ \\
\hline $5.2-5.4$ & $7.81707 \pm 0.00041$ & $-108.4015 \pm 0.0639$ & $100.7171 \pm 0.0261$ & $-39.86233 \pm 0.00308$ \\
\hline $5.4-5.6$ & $8.19870 \pm 0.00046$ & $-126.8290 \pm 0.0774$ & $108.3524 \pm 0.0304$ & $-42.78088 \pm 0.00348$ \\
\hline $5.6-5.8$ & $8.58279 \pm 0.00051$ & $-147.3051 \pm 0.0940$ & $116.3804 \pm \mathbf{0 . 0 3 5 7}$ & $-45.81359 \pm 0.00393$ \\
\hline $5.8-6.0$ & $8.96735 \pm 0.00057$ & $-169.9489 \pm 0.1139$ & $124.6019 \pm 0.0418$ & $-48.94595 \pm 0.00444$ \\
\hline $6.0-6.2$ & $9.35345 \pm 0.00063$ & $-194.3185 \pm 0.1394$ & $133.1162 \pm 0.0492$ & $-52.18043 \pm 0.00502$ \\
\hline $6.2-6.4$ & $9.73840 \pm 0.00069$ & $-220.7647 \pm 0.1712$ & $141.9239 \pm 0.0578$ & $-55.51927 \pm 0.00565$ \\
\hline $6.4-6.6$ & $10.12738 \pm 0.00077$ & $-249.7792 \pm 0.2091$ & $151.0865 \pm 0.0677$ & $-58.96573 \pm 0.00638$ \\
\hline $6.6-6.8$ & $10.51659 \pm 0.00085$ & $-281.1153 \pm 0.2561$ & $160.3162 \pm 0.0802$ & $-62.52332 \pm 0.00736$ \\
\hline $6.8-7.0$ & $10.90541 \pm 0.00094$ & $-315.0544 \pm 0.3112$ & $170.1481 \pm 0.0941$ & $-66.16998 \pm 0.00829$ \\
\hline $7.0-7.2$ & $11.29740 \pm 0.00104$ & $-352.3613 \pm 0.3820$ & $180.2278 \pm 0.1102$ & $-69.93934 \pm 0.00915$ \\
\hline $7.2-7.4$ & $11.68581 \pm 0.00115$ & $-390.4128 \pm 0.4679$ & $189.9568 \pm 0.1295$ & $-73.79687 \pm 0.01033$ \\
\hline $7.4-7.6$ & $12.08266 \pm 0.00128$ & $-432.7892 \pm 0.5839$ & $200.9453 \pm 0.1531$ & $-77.80139 \pm 0.01165$ \\
\hline $7.6-7.8$ & $12.47254 \pm 0$ & $-478.4916 \pm 0.7052$ & $211.5939 \pm 0.1792$ & $-81.87225 \pm 0.01313$ \\
\hline $7.8-8.0$ & $12.86991 \pm 0.00156$ & $-524.9324 \pm 0.9245$ & $223.0187 \pm 0.2138$ & $-86.09206 \pm 0.01483$ \\
\hline $8.0-8.2$ & $13.26321 \pm 0.00172$ & $-577.0701 \pm 1.0982$ & $234.3402 \pm 0.2533$ & $-90.38641 \pm 0.01671$ \\
\hline $8.2-8.4$ & $13.65319 \pm 0.00190$ & $-628.5931 \pm 1.4289$ & $245.7515 \pm 0.3025$ & $-94.76859 \pm 0.01887$ \\
\hline $8.4-8.6$ & $14.05098 \pm 0.00211$ & $-686.1360 \pm 1.7585$ & $257.7179 \pm 0.3484$ & $-99.29108 \pm 0.02128$ \\
\hline $8.6-8.8$ & $14.44353 \pm 0.00232$ & $-750.9577 \pm 1.8829$ & $270.1815 \pm 0.4105$ & $-103.89006 \pm 0.02399$ \\
\hline $8.8-9.0$ & $14.83704 \pm 0.00257$ & $-815.3002 \pm 2.3285$ & $283.1744 \pm 0.4893$ & $-108.57330 \pm 0.02705$ \\
\hline $9.0-9.2$ & $15.23688 \pm 0.00283$ & $-877.7905 \pm 3.2236$ & $296.2676 \pm 0.6484$ & $-113.44241 \pm 0.03055$ \\
\hline $9.2-9.4$ & $15.63060 \pm 0.00313$ & $-954.9899 \pm 3.8191$ & $308.4226 \pm 0.7593$ & $-118.36857 \pm 0.03441$ \\
\hline $9.4-9.6$ & $16.03193 \pm 0.00346$ & $-1030.7033 \pm 4.4742$ & $322.0296 \pm 0.8728$ & $-123.46222 \pm 0.03884$ \\
\hline $9.6-9.8$ & $16.42336 \pm 0.00383$ & $-1108.4613 \pm 4.8676$ & $336.9731 \pm 0.9487$ & $-128.52130 \pm 0.04392$ \\
\hline $9.8-10.0$ & $16.82386 \pm 0.00423$ & $-1188.7666 \pm 8.4394$ & $350.7993 \pm 1.3343$ & $-133.89305 \pm 0.04955$ \\
\hline $10.0-10.2$ & $17.22008 \pm 0.00467$ & & & $-139.24776 \pm 0.05602$ \\
\hline $10.2-10.4$ & $17.61896 \pm 0.00517$ & & & $-144.67924 \pm 0.06332$ \\
\hline $10.4-10.6$ & $18.01720 \pm 0.00571$ & & & $-150.24974 \pm 0.07139$ \\
\hline $10.6-10.8$ & $18.41918 \pm 0.00631$ & & & $-156.05150 \pm 0.08068$ \\
\hline $10.8-11.0$ & $18.81383 \pm 0.00695$ & & & $-161.75693 \pm 0.09050$ \\
\hline $11.0-11.2$ & $19.22148 \pm 0.00771$ & & & $-167.73174 \pm 0.10267$ \\
\hline $11.2-11.4$ & $19.60535 \pm 0.00848$ & & & $-173.55084 \pm 0.11589$ \\
\hline $11.4-11.6$ & $20.00483 \pm 0.00939$ & & & $-179.76825 \pm 0.13097$ \\
\hline $11.6-11.8$ & $20.40568 \pm 0.01040$ & & & $-185.96573 \pm 0.14723$ \\
\hline $11.8-12.0$ & $20.82530 \pm 0.01146$ & & & $-192.55302 \pm 0.16694$ \\
\hline
\end{tabular}

Table 2: Monte Carlo results for coefficients $a, b_{F}, b_{A}$ and $b_{T}$. 

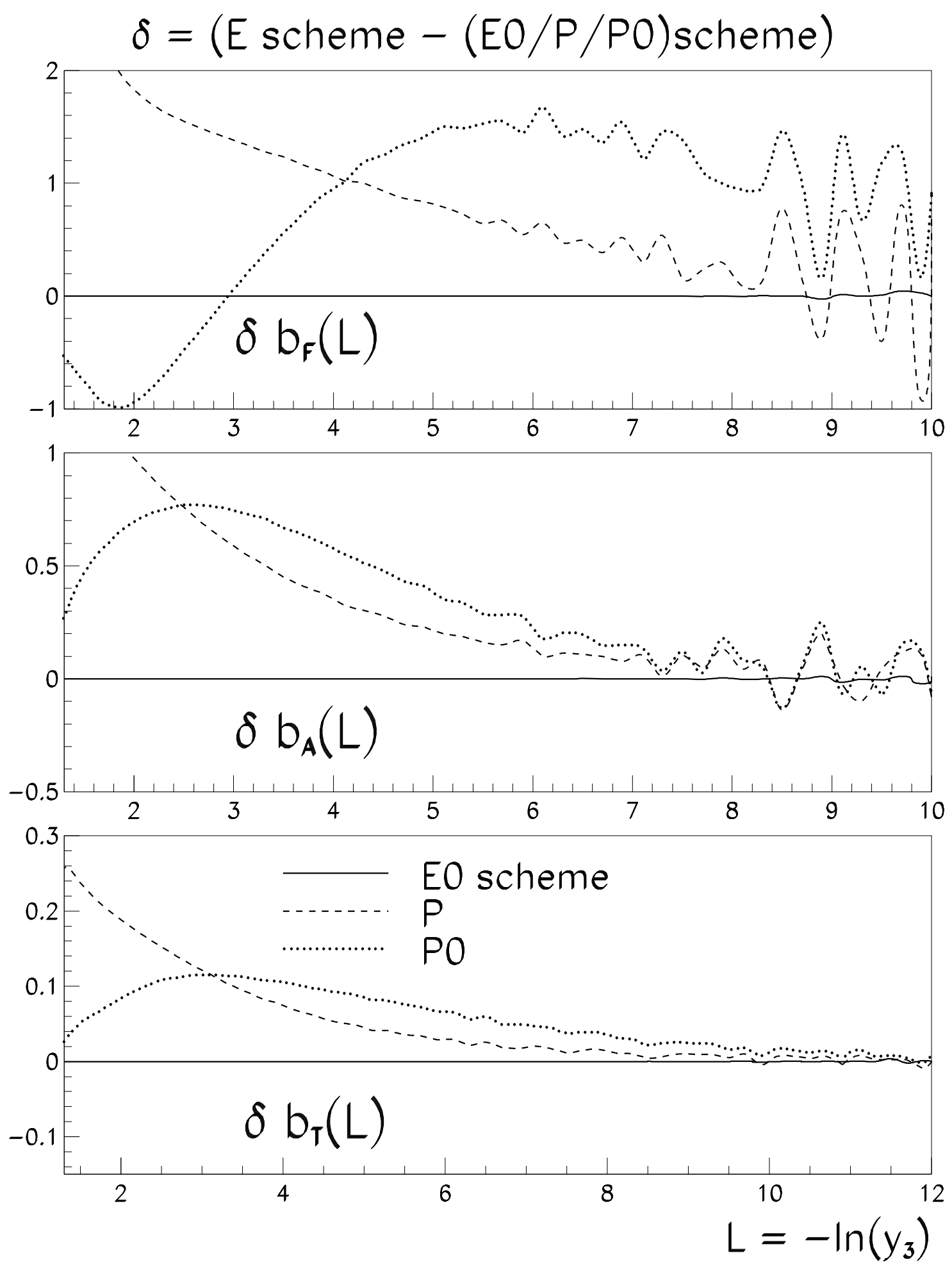

Figure 1: Absolute differences between the coefficients $b_{*}(L)$ obtained from a Monte Carlo integration for the various recombination schemes. 

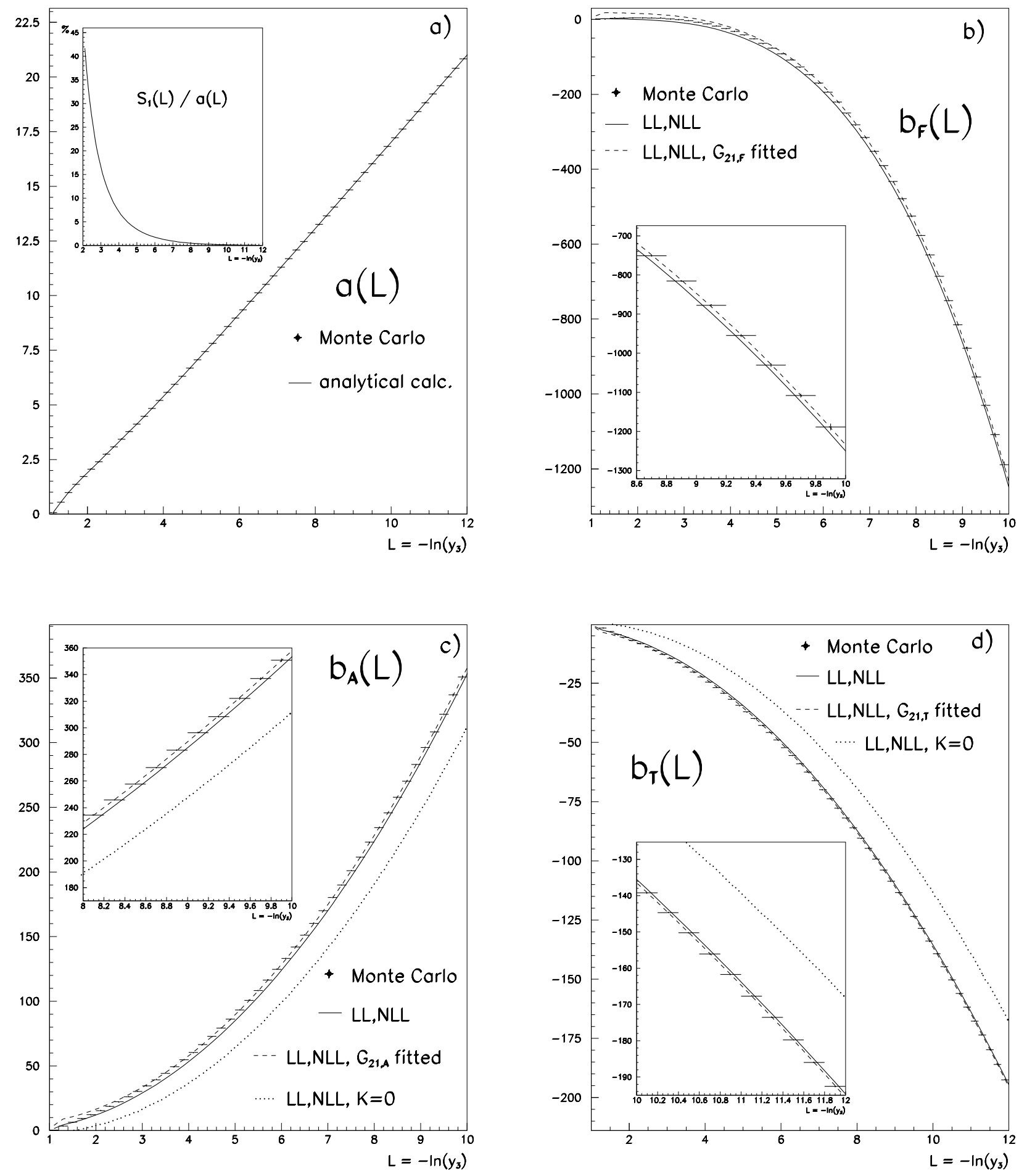

Figure 2: a) First order coefficient $a(L)$ obtained by Monte Carlo compared to the analytical result. The inset shows the relative contribution of the non-logarithmic terms to the full first order prediction. b-d) Second order coefficients $b_{*}(L)$. Plotted are the Monte Carlo result, the predictions obtained from all known LL and NLL terms, the (LL,NLL) predictions improved by the addition of the fitted subleading coefficients $G_{21, *}$ and the incomplete (LL,NLL) predictions $(K=0)$. 

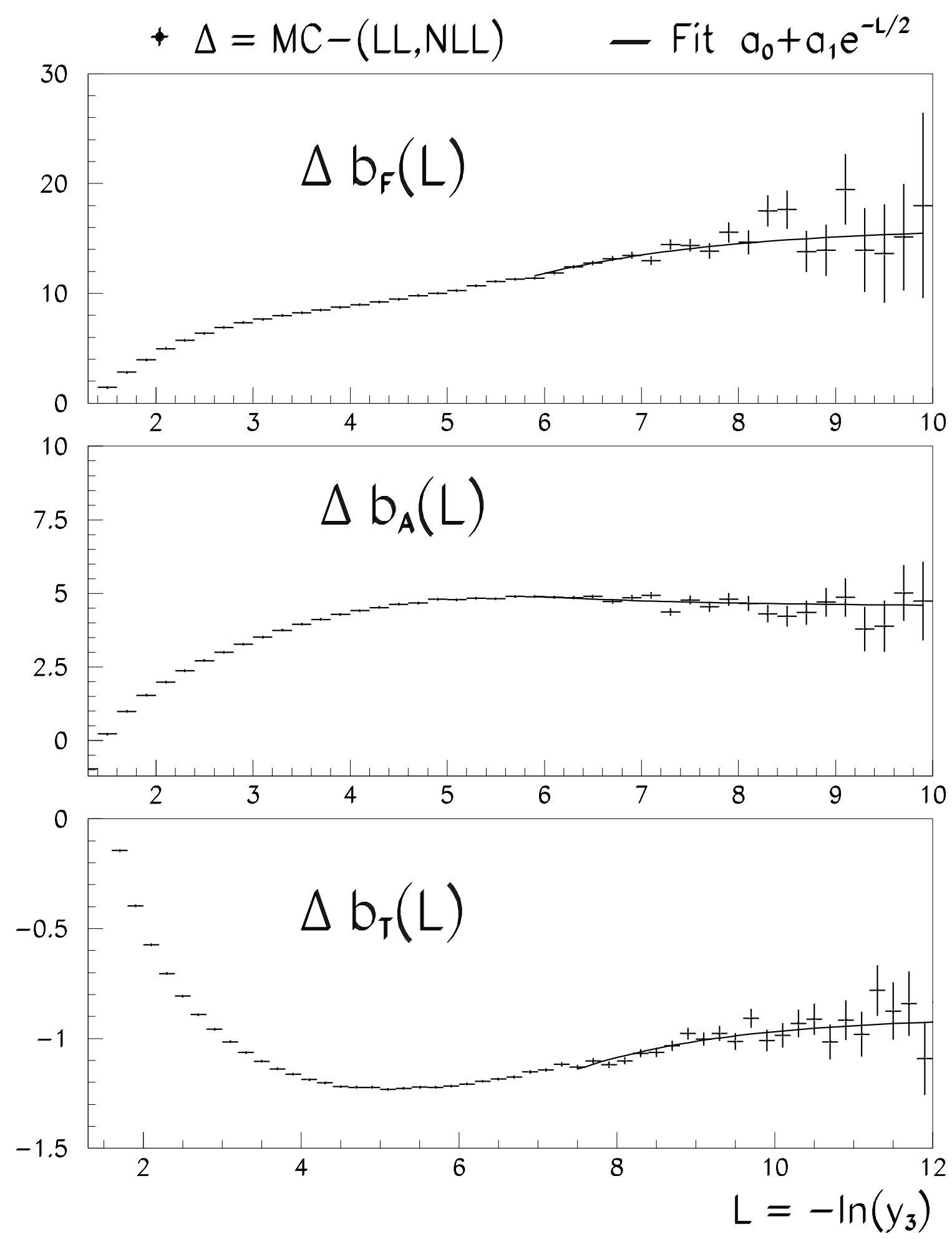

Figure 3: Differences between the Monte Carlo integration and all known LL and NLL terms for the second order coefficients $b_{*}(L)$. The lines show the fitted functions $a_{0}+a_{1} e^{-L / 2}$ over the range used in the fit. 


\section{Second order $Q C D$ coefficient of $R_{2}(L)$}

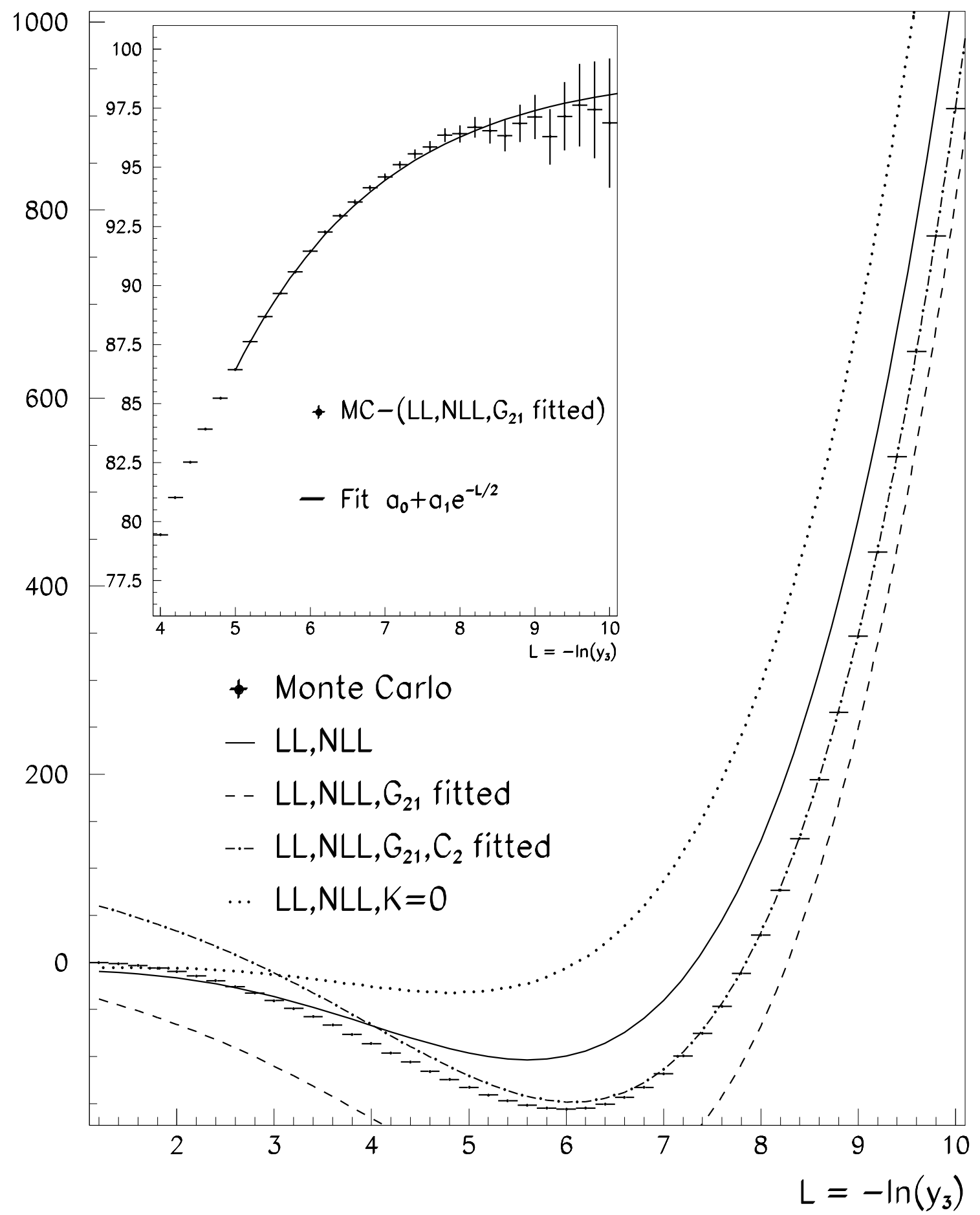

Figure 4: The second order coefficient of the cumulative 2-jet rate. Plotted are the Monte Carlo result and variations of the (LL,NLL) calculation, taking into account also the known subleading coefficient $C_{1}$. The inset shows the difference between the Monte Carlo integration and all known LL,NLL and subleading terms, as well as a fit of its asymptotic tail. 